\title{
Highly anisotropic crosslinked HDPE foams with a controlled anisotropy ratio: Production and characterization of the cellular structure and mechanical properties
}

\author{
Victoria Bernardo ${ }^{\mathrm{a}, *}$, Ester Laguna-Gutierrez ${ }^{\mathrm{a}}$, Alberto Lopez-Gil ${ }^{\mathrm{b}}$, Miguel Angel Rodriguez-Perez ${ }^{\mathrm{a}}$ \\ a Cellular Materials Laboratory (CellMat), Condensed Matter Physics Department, University of Valladolid, Valladolid, Spain \\ b CellMat Technologies, Valladolid, Spain
}

\section{H I G H L I G H T S}

- Highly anisotropic high density polyethylene foams, with anisotropy ratios as high as 3.75 , have been produced.

- The two-step foaming process used allows controlling the anisotropy ratio by changing the temperature in the first step.

- This method allows producing highly anisotropic foams in complex shapes by means of the control of the process parameters.

\section{G R A P H I C A L A B S T R A C T}

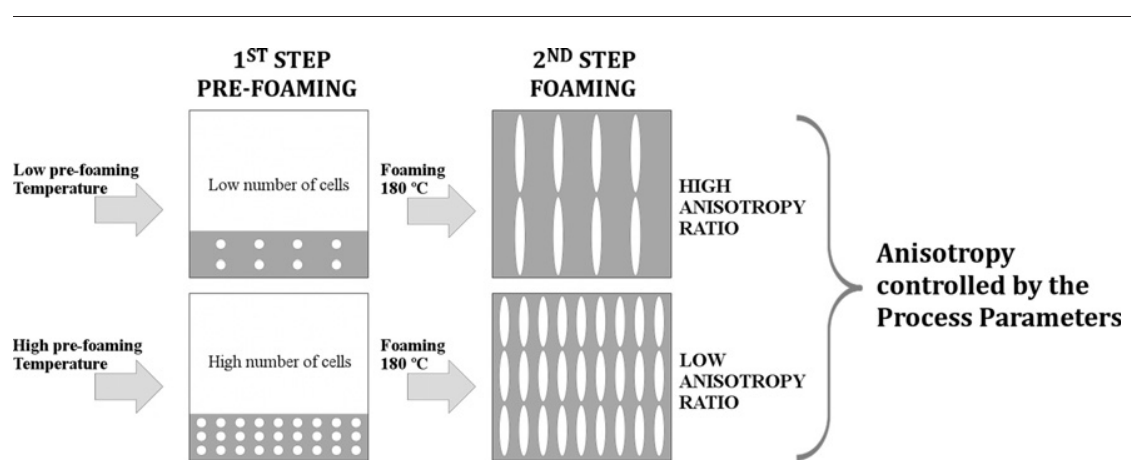

\begin{abstract}
A B S T R A C T
In this paper a two-step foaming process was used to produce highly anisotropic foams with controlled anisotropy ratio. Anisotropic cellular materials are of great interest due to the enhanced mechanical properties that they show in the anisotropy direction. Therefore, the production of foams with high and controlled anisotropy ratios is an essential topic when it comes to design materials with good mechanical performance. In this work, high density polyethylene (HDPE) foams with anisotropy ratios larger than 3 have been produced. A foaming process consisting of two consecutive heating steps: pre-foaming and foaming, has been employed. Foaming kinetics at four different pre-foaming temperatures $\left(145^{\circ} \mathrm{C}, 155^{\circ} \mathrm{C}, 170^{\circ} \mathrm{C}\right.$ and $\left.180^{\circ} \mathrm{C}\right)$ and at a constant foaming temperature $\left(180^{\circ} \mathrm{C}\right)$ have been performed. Results showed that the pre-foaming temperature is the key parameter that allows controlling the anisotropy ratio of the final foam. A relationship between the pre-foaming temperature, the number of cells and the anisotropy ratio has been established. Low pre-foaming temperatures lead to low numbers of cells, which eventually results in high anisotropy ratios. Mechanical properties in compression were measured and compared with the anisotropy of the cellular structure, being that the elastic modulus increases with the anisotropy ratio.
\end{abstract}

(c) 2016 Elsevier Ltd. All rights reserved.

\footnotetext{
* Corresponding author at: Paseo de Belén 7, 47011 Valladolid, Spain.

E-mail addresses: vbernardo@fmc.uva.es (V. Bernardo), ester.laguna@fmc.uva.es (E. Laguna-Gutierrez), a.lopez@cellmattechnologies.com (A. Lopez-Gil), marrod@fmc.uva.es (M.A. Rodriguez-Perez).
}

\section{Introduction}

Polymeric cellular materials are defined as two-phase systems in which a gaseous phase is dispersed in a continuous solid polymeric matrix [1]. The properties of these materials are conditioned by the properties of the solid polymer matrix and by the properties associated to the 
fact of having a cellular structure. Polymeric cellular materials are commonly used in applications such as packing, cushioning, automotive and thermal insulation [2]. The aforementioned applications require lightweight materials; however, mechanical behavior is also a key factor, which determines the final use of polymer foams. For example, for packing applications materials with high energy-absorption capacity are demanded $[3,4]$.

In that aspect, high density polyethylene (HDPE) is a polymer with excellent mechanical properties. This polymer is tough, stiff, chemical and abrasion resistant and presents a low absorption and permeability to water and moisture [5-7]. Moreover, HDPE can withstand higher temperatures than other polymers, which is essential for some applications. The foams based on HDPE inherit these properties and hence, these materials are very interesting for a wide range of applications, from structural foams [8-10] to fillers for lighting cement mortars [11].

However, it is well known that for foams the loss of mechanical properties at relative low densities is very significant, with normally a square dependency with density [2]. Therefore, an important part of the research on cellular materials is focused on studying and developing different strategies that could be followed to increase the mechanical properties of these materials at a given density [12].

There are several factors that influence the mechanical response of foams. On the one hand, foam density is the main factor that determines the mechanical behavior of cellular materials [2,12-14]. On the other hand, mechanical properties are also influenced by the characteristics of the cellular structure. For instance, high open cell contents, that is, high interconnectivities between cells, are not desirable since both elastic modulus and collapse stress decrease as the open cell content increases $[14,15]$. The homogeneity of the cellular structure is also a factor to consider [16]. The heterogeneous cell size distributions lead to lower mechanical properties than the homogeneous ones [12]. Shape and size of the cells also influence the properties of these materials. While the effect of the cell size in conventional foams is not clearly established [17], the anisotropy of the cellular structure has been proved to have a strong influence in the mechanical behavior [2,18-21].

Anisotropic cellular structures are characterized by being composed of cells elongated in one direction. Cell anisotropy can be quantified through the anisotropy ratio, which is defined as the ratio between the maximum and the minimum length of the cell [22]. Anisotropic foams present an improvement in the compression modulus along the anisotropy direction. According to the model of Gibson and Ashby [12], the modulus of the foam, $E_{f}$, is proportional to its anisotropy ratio, $R$, by Eq. (1), where $E_{s}$ is the modulus of the solid, $C$ is a constant, $n$ is the exponent which takes into account the cellular structure, $\rho_{f}$ is the density of the foam and $\rho_{s}$ is the density of the solid. This means that an increase of a factor of 2 in the anisotropy ratio would lead to a double modulus if the relative density $\left(\rho_{f} / \rho_{s}\right)$ is the same. In addition, the ratio between the modulus in the anisotropy direction $(\mathrm{y})$ and in a direction perpendicular to the first one $(\mathrm{x}), E_{y} / E_{x}$, can be expressed a function of $R$, as described by Eq. (2).

$\frac{E_{f}}{E_{s}}=C\left(\frac{\rho_{f}}{\rho_{s}}\right)^{n} R$

$\frac{E_{y}}{E_{x}}=\frac{2 R^{2}}{1+\left(\frac{1}{R^{3}}\right)}$

Shear resistance and fracture toughness are sensitive to anisotropy in cell shape as well $[12,22]$. In particular, the ratio of the shear modulus in the anisotropy direction $\left(G_{y}\right)$ and in the direction perpendicular to it $\left(G_{x}\right)$ decreases with anisotropy according to Eq. (3).

$\frac{\mathrm{G}_{\mathrm{y}}}{\mathrm{G}_{\mathrm{x}}}=\frac{2}{1+\mathrm{R}}$
These enhanced mechanical properties make anisotropic foams suitable for some specific applications were outstanding properties are demanded. For instance, anisotropic foams have been proved to be adequate for head impact protection, as the reduced shear resistance of these foams results in a reduced transmission of transverse forces to the head [23-25]. Besides, anisotropic foams can be good candidates for the foamed core of sandwich panels [26,27]. Sandwich panels are used in a broad market sector. They are employed in the manufacturing of hull of yachts or roofs of trains. For these applications light-weight materials are required, but good mechanical performance is needed as well. In this sense, anisotropic foams present a solution for improving the mechanical properties at the same density.

Thermal properties also depend on the anisotropy of the cellular structure $[28,29]$. Thermal conduction through the solid phase, $\lambda_{s}$, depends on the anisotropy ratio according to Eq. (4), where $f_{s}$ is the fraction of mass in the struts and $\lambda_{p}$ is the thermal conductivity of the polymer. Thermal conductivity is larger in the anisotropy direction, and this property makes anisotropic foams suitable for specific applications requiring low density foams with low thermal insulation capacity. Foams usually present low thermal conductivities, so in this aspect anisotropic foams may extend the range of properties of cellular materials with higher thermal conductivities in the anisotropy direction.

$\lambda_{s}=\frac{1}{3}\left(\frac{\rho_{f}}{\rho_{s}}\right) f_{s} \lambda_{p} \sqrt{R}$

According to Eqs. (1)-(4), foams with tailor-made physical properties can be obtained just by adjusting the anisotropy ratio of the cellular structure. Polymer foams usually present anisotropy ratios of about 1.3 [3]. To obtain larger anisotropy ratios, processes designed specifically are required. In the work of Laguna-Gutierrez et al. [30] the improve compression molding (ICM) technique was employed to produce polypropylene foams with controlled expansion ratios of the order of 5 and anisotropy ratios around 3. In the work of Arora et al. [31] anisotropic polystyrene foams were produced using supercritical carbon dioxide as blowing agent. In this case the cell shape was controlled by means of the vessel in which the sample is foamed, as its size can constrain the growth of the foam. Another different strategy was followed by Oliveira-Salmazo et al. [32]. They found a way to vary the anisotropy ratio in natural rubber foams by changing the dimensions of the solid precursors. Expansion ratios around 3 and anisotropy ratios between 0.9 and 2.48 were obtained. Kleiner et al. [33] prepared crosslinking polyethylene foam layers with an anisotropic structure. For this purpose, they prepared layers with different blowing agent contents. The layers were then arranged as a multilayer structure so that the two external layers had a blowing agent content smaller than the internal one. The composed material was put under temperature and an anisotropic structure was obtained in the internal layer, being the rest of the sample isotropic.

The strategy employed to increase the anisotropy ratio of polymeric foams consists on restricting the cell growth to one direction either through a mold or through multiphase structures with different compositions. As far as the author knows, there are no works in which the process parameters are used to control the foam anisotropy ratio. The main objective of this work is to develop a new production route that allows controlling cell anisotropy by means of modifying the process parameters, and not by using a mechanical process or materials with different chemical compositions. Since only the process parameters determine the final anisotropy ratio, this method will allow producing highly anisotropy foams with controlled anisotropy ratio using the same raw material and the same mold. Crosslinked HDPE foams have been produced using this innovative method and the relationship between the process parameters, the structure and the mechanical properties of the foams has been studied. This paper also aims at understanding the physical origin of the different anisotropic structures observed. 


\section{Experimental}

\subsection{Materials}

The polymer matrix used was HDPE Rigidex® HD5226EA supplied by INEOS POLYOLEFINS, with melting point of $131{ }^{\circ} \mathrm{C}$, density of $0.953 \mathrm{~g} / \mathrm{cm}^{3}$ and melt flow index of $26 \mathrm{~g} / 10 \mathrm{~min}$ (measured at $190{ }^{\circ} \mathrm{C}$ and $2.16 \mathrm{~kg}$ ). The crosslinking agent was dicumyl peroxide (DCP) Luperox DC40P supplied by ARKEMA. Azodicarbonamide (ADC) Unifoam AZ VI-50 supplied by HEBRON S.A. was employed as blowing agent. It has a decomposition temperature of $200{ }^{\circ} \mathrm{C}$ and an average particle size of $20 \mu \mathrm{m}$. Other additives were used too: a lubricant agent, stearic acid supplied by RENICHEM, and an antioxidant, Irganox 1010 supplied by CIBA, both in powder form. All the data of the raw materials were obtained from their respective technical data sheets.

\subsection{Samples preparation}

The formulation described in Table 1 was produced using a twinscrew extruder, model COLLIN TEACH-LINE ZK 25T, with L/D of 24 and screw diameter of $25 \mathrm{~mm}$. The temperature profile set on the extruder was from $110{ }^{\circ} \mathrm{C}$ to $130{ }^{\circ} \mathrm{C}$ in the die, increasing in intervals of $5{ }^{\circ} \mathrm{C}$. The screw speed was $60 \mathrm{rpm}$. Low temperatures and low feeding rates were employed to avoid premature decomposition of both crosslinking and blowing agents.

The molten was cooled in a water bath and pelletized. After a drying of $24 \mathrm{~h}$ in a vacuum oven at $60^{\circ} \mathrm{C}$, the material was extruded again with the aim of dispersing homogenously all the additives. The temperature profile and the screw speed remained the same. Several thermogravimetric analyses (TGA) of the final formulation were performed to confirm its homogeneity.

\subsection{Foaming process}

The formulation previously described was foamed using an aluminum cylindrical mold with a mold cavity of $24.9 \mathrm{~cm}^{3}$. The heat required for the foaming process was transferred by a resistance clamp as shown in Fig. 1. Temperature was controlled by using a thermocouple located inside the upper cover of the mold. The material, in pellet form, was introduced in the mold. A constant mass $(3.12 \mathrm{~g}$ ) was always used with the aim of producing foams with the same density. The introduction of the pellets in the mold was performed with the mold at room temperature.

The foaming route selected to produce the foams consisted of two main steps, as indicated in Fig. 2:

1. Pre-foaming: The material was heated at a temperature above the DCP decomposition temperature and it was held at that temperature for $15 \mathrm{~min}$. During this step a pre-expansion is obtained but also a crosslinking of the polymer matrix. Four different temperatures were employed $\left(145^{\circ} \mathrm{C}, 155^{\circ} \mathrm{C}, 170{ }^{\circ} \mathrm{C}\right.$ and $\left.180^{\circ} \mathrm{C}\right)$ so as to obtain four different crosslinking and pre-expansion degrees before the foaming step.

2. Foaming: The temperature was raised up to $180^{\circ} \mathrm{C}$ to fully release the blowing agent. The evolution of the foaming process was evaluated by producing foams with different foaming times: from 0 to $30 \mathrm{~min}$

Table 1

Formulation.

\begin{tabular}{ll}
\hline Raw material & $\mathrm{phr}^{\mathrm{a}}$ \\
\hline High density polyethylene (HDPE) & 100 \\
Dicumyl peroxide (DCP) & 6.2 \\
Azodicarbonamide (ADC) & 18.5 \\
Steric acid & 1.2 \\
Antioxidant & 1.2 \\
\hline
\end{tabular}

a Parts per hundred of polyethylene.

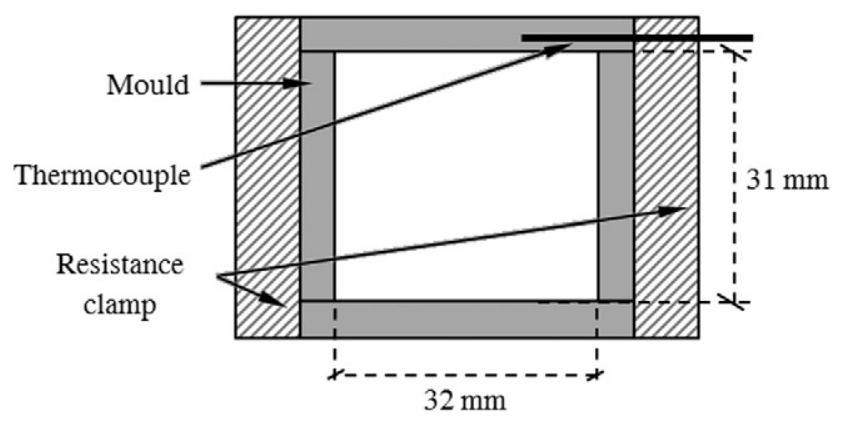

FIG. 1. Cross section of the set up used to produce the foams.

at intervals of $5 \mathrm{~min}$. After the appropriate foaming time the mold was cooled in a water bath to stabilize the cellular structure as fast as possible. The dots in Fig. 2 indicate the times at which the foams were produced.

Foaming kinetics were studied for the four different pre-foaming temperatures. The terminology chosen for the four material sets is the following: PF-145, PF-155, PF-170 and PF-180, where PF comes from pre-foaming and the number indicates the temperature in ${ }^{\circ} \mathrm{C}$ of this step.

\subsection{Samples characterization}

\subsubsection{Density}

Density of the pelletized solid material was measured with a gas pycnometer, Micromeritics AccuPyc II 1340. For the foamed materials density was determined by the geometric method; that is, the weight of each foam was divided by its corresponding volume, according with ASTM standard D1622-08.

\subsubsection{Cellular structure}

Optical images were used for studying the cellular structure of the foamed samples. The cylindrical foams were cut with a razor blade in two parts following the growing direction in order to obtain a view of the plane $x y$, where $y$ is the expansion direction and $x$ is a perpendicular direction to the expansion one. As the expansion was isotropic in any plane perpendicular to the $y$ axis, the election of the $x$ axis was arbitrary.

Images of the aforementioned plane were taken using an optical digital camera. The cells were stood out with ink to obtain an image in which binarization is more precise. The images were analyzed by using an image processing analysis tool based on the software Fiji/

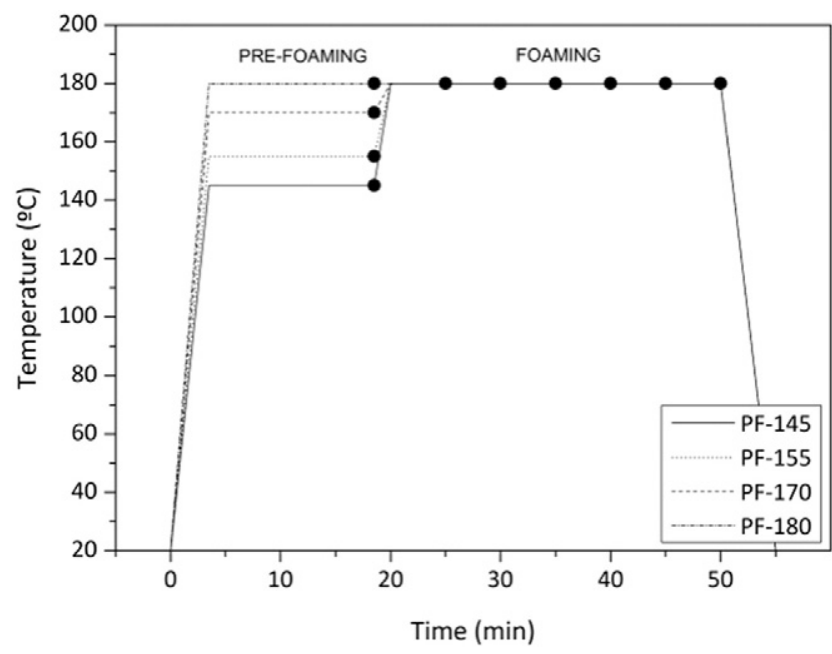

Fig. 2. Temperature profiles for the four series of foams produced. 

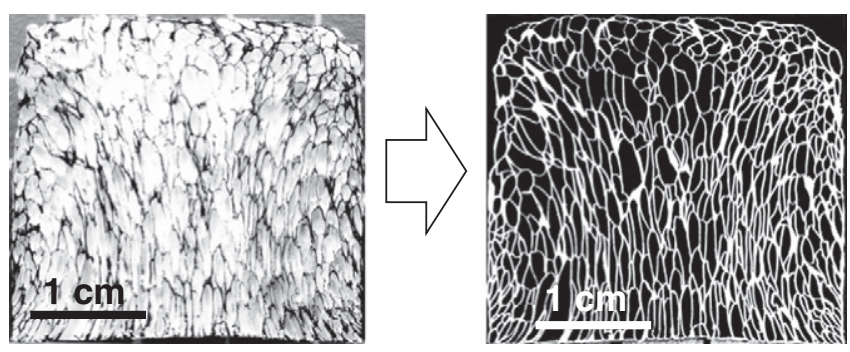

Fig. 3. Optical images before and after binarization.

Image J [34]. The first step of the image analysis was the binarization of the images as shown in Fig. 3.

In a second step several cellular structure parameters were determined. One of the aims of this work is to study the evolution of the number of cells during the foaming process. Therefore, the complete image was used in order to count all the cells that are located in the selected area $\left(N_{2 D}\right)$. Nevertheless, there are some defaults in the foams. First, the cells on the sides are deformed because the material adheres to the walls of the mold. Second, some cells are deformed when the foam reaches the upper cover of the mold. This is due to the pressure of the cells located below which continue growing after filling the mold. These defects can be seen in Fig. 3. In order to avoid these issues, a second analysis of the images was performed, reducing the area a $10 \%$ on each lateral side and a $10 \%$ on the upper part. Despite the reduced area, a significant part of the cells (more than 50\%) was considered for the structural analysis.

After doing this second analysis, cell sizes in both $x$ and $y$ directions ( $\phi_{x}$ and $\phi_{y}$ respectively) were determined. From these values the anisotropy ratio $(R)$ was calculated using Eq. (5).

$\mathrm{R}=\frac{\phi_{\mathrm{y}}}{\phi_{\mathrm{x}}}$

The cell size in four directions $\left(0^{\circ}, 45^{\circ}, 90^{\circ}\right.$ and $135^{\circ}$, being $0^{\circ}$ the $x$ axis) were evaluated. From these measurements it was possible to determine the direction in which the cells are larger, that is, the orientation of the cells $(\theta)$. Furthermore, the cell density, defined as the number of cells per unit of volume of the foamed material $\left(N_{v}\right)$, was calculated using Eq. (6):

$\mathrm{N}_{\mathrm{v}}=\left[\frac{\mathrm{nM}^{2}}{\mathrm{~A}}\right]^{\frac{3}{2}}$

where $n$ is the number of cells analyzed, $A$ is the image area and $M$ is the magnification factor of the micrograph. This calculus is based on the Kumar's theoretical approximation [17,34,35].

Assuming that cells are ellipsoids of revolution with two equal axes $\left(\phi_{x}\right)$ and one different $\left(\phi_{y}\right)$, the average three dimensional cell size $\left(\phi_{3 D}\right)$ was calculated according to Eq. (7). Notice the three dimensional correction factor 1.273 [34], which is necessary to calculate a three dimensional magnitude from the two dimensional values $\phi_{x}$ and $\phi_{y}$.

$\phi_{3 \mathrm{D}}=1.273\left(\frac{2 \phi_{\mathrm{x}}+\phi_{\mathrm{y}}}{3}\right)$

\subsubsection{Gel content}

The gel content (GC) produced by crosslinking was determined according to the standard ASTM D 2765-01. Specimens of the crosslinked samples were first weighed and then immersed in boiling xylene, which was used as extracting solvent. Samples were kept in boiling xylene for $1 \mathrm{~h}$, which is the minimum time required to dissolve completely the uncrosslinked HDPE. After this time, samples were removed from xylene and dried in a vacuum oven pre-heated at $150{ }^{\circ} \mathrm{C}$. Finally, the specimens were reweighed to determine the insoluble fraction of polymer and therefore, the gel content according to Eq. (8), where $W_{f}$ is the weight of the specimen after extraction and drying and $W_{0}$ is the weight of the original specimen. Three samples were used to determine the average gel content for each material.

$$
\mathrm{GC}(\%)=100\left(1-\frac{\mathrm{W}_{0}-\mathrm{W}_{\mathrm{f}}}{\mathrm{W}_{0}}\right)
$$

a)

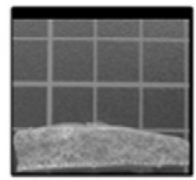

b)

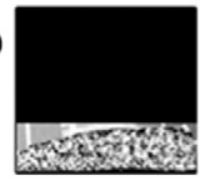

c)

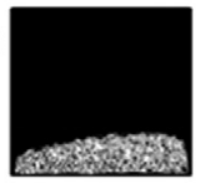

d)

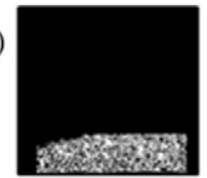

$0 \mathrm{~min}$
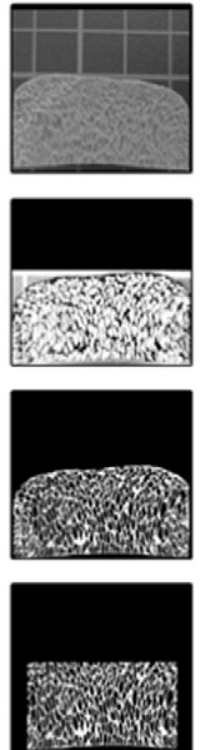

$5 \mathrm{~min}$
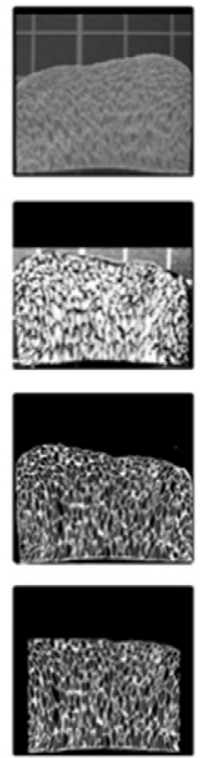
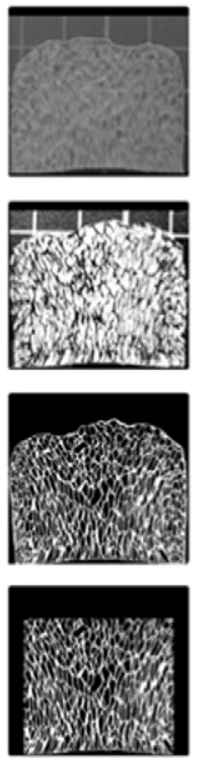

$15 \mathrm{~min}$
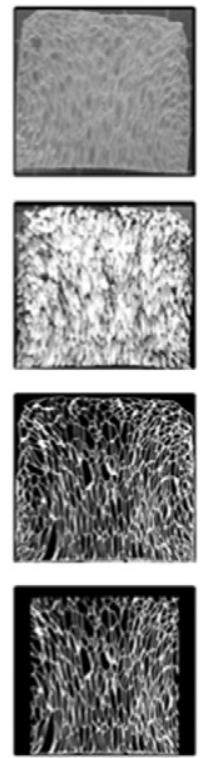

$20 \mathrm{~min}$
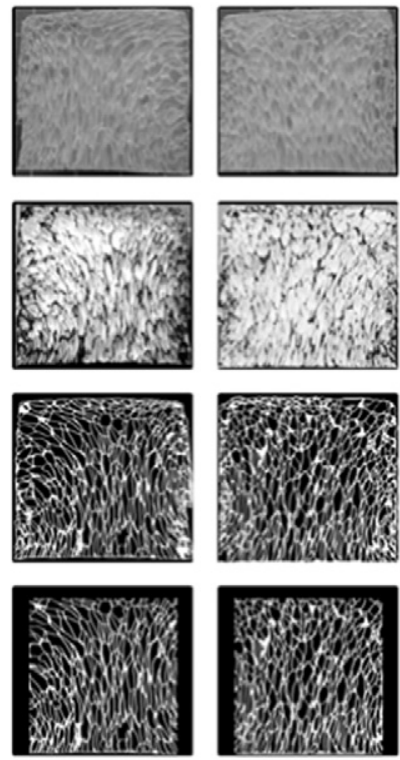

$25 \mathrm{~min}$

Foaming Time

Fig. 4. Foaming kinetics for the PF-155 series. a) Original image. b) Cells stood out. c) Binarization. d) Images cut. 


\subsubsection{Blowing agent content}

Both the initial amount of blowing agent and the percentage released after the pre-foaming step were determined by thermogravimetric analysis in a Mettler TGA/SDTA 851 equipment. For measuring the initial amount of blowing agent samples were heated from $50{ }^{\circ} \mathrm{C}$ to $850{ }^{\circ} \mathrm{C}$ at a heating rate of $20^{\circ} \mathrm{C} / \mathrm{min}$ under nitrogen atmosphere. The amount of blowing agent released after the pre-foaming step was determined by performing a thermogravimetric analysis at the pre-foaming temperature $\left(145^{\circ} \mathrm{C}, 155^{\circ} \mathrm{C}, 170^{\circ} \mathrm{C}\right.$ or $\left.180^{\circ} \mathrm{C}\right)$ during $15 \mathrm{~min}$.

\subsubsection{Mechanical tests}

Mechanical properties in compression have been measured with a Universal Testing Machine INSTRON (model 5.500R6025). Stressstrain curves were obtained at room temperature and at a strain rate of $10 \mathrm{~mm} / \mathrm{min}$. The maximum static strain was $75 \%$, for all the experiments. The compression experiments were performed according to the standard ASTM D1621. The test samples were cubes of $10 \mathrm{~mm}$ taken from the center of the foams. The stress was applied in the $y$ direction (anisotropic direction) and the elastic modulus $(E)$ was calculated as the slope of the stress-strain curves in the linear elastic region.

\section{Results}

\subsection{Analysis of the foaming kinetics}

One of the foaming kinetics (PF-155) studies is shown in Fig. 4. The first row (Fig. 4.a) contains the images of the original foams taken in the $x y$ plane. The other three rows represent the steps followed during the image analysis process: the cells stood out (Fig. 4.b), the binarization (Fig. 4.c) and finally the cut of the images in order to have a representative area without surface defects (Fig. 4.d).

These images show the evolution of the foaming process with the foaming time. It is important to remark that, as expected, at the end of the pre-foaming time (foaming time equal to zero) a cellular structure is already observed. This means that part of the ADC is decomposed during the pre-foaming, even for low pre-foaming temperatures. Besides, the image of the kinetics shows the growth of the foam during the process. In the case of PF-155, after 20 min the foam reaches the upper cover of the mold, that is, the material reaches the maximum expansion. The other kinetics are similar: all the materials present cells at foaming time equal to zero and the maximum expansion is reached after a certain time. The first part of the foaming process, which corresponds to the expansion up to filling the mold, will be called growing stage from now on; the time after filling the mold will be denoted as ageing stage.

\subsubsection{Density}

Fig. 5 shows the cellular materials density as a function of the foaming time. The two aforementioned stages are perfectly distinguished in this plot. During the growing stage (open symbols) the foams density decreases because the materials are still expanding. In the last point of this stage, density reaches a minimum value, which indicates that the mold is filled. Then, during the ageing stage (closed symbols) the foams density remains constant. Final density for all series is around $140 \pm 2 \mathrm{~kg} / \mathrm{m}^{3}$.As the density of the solid material was $1039.7 \pm 0.4 \mathrm{~kg} / \mathrm{m}^{3}$, the expansion ratio of the final foams is close to 7.5 times.

It is important to remark that the time at which the minimum density is achieved is different for each series. The PF-145 and the PF-155 series need 20 min of foaming time to reach the maximum expansion. The PF-170 series needs only $15 \mathrm{~min}$ and this time is reduced up to 5 min for the PF-180 series. The expansion starts during the prefoaming step; therefore, a higher temperature in this step leads to a sample with a lower density at the beginning of the foaming step (foaming time equal to zero). Thus, the foaming time required to fill the mold decreases as the pre-foaming temperature increases. These times at which the expansion reaches it maximum value can be

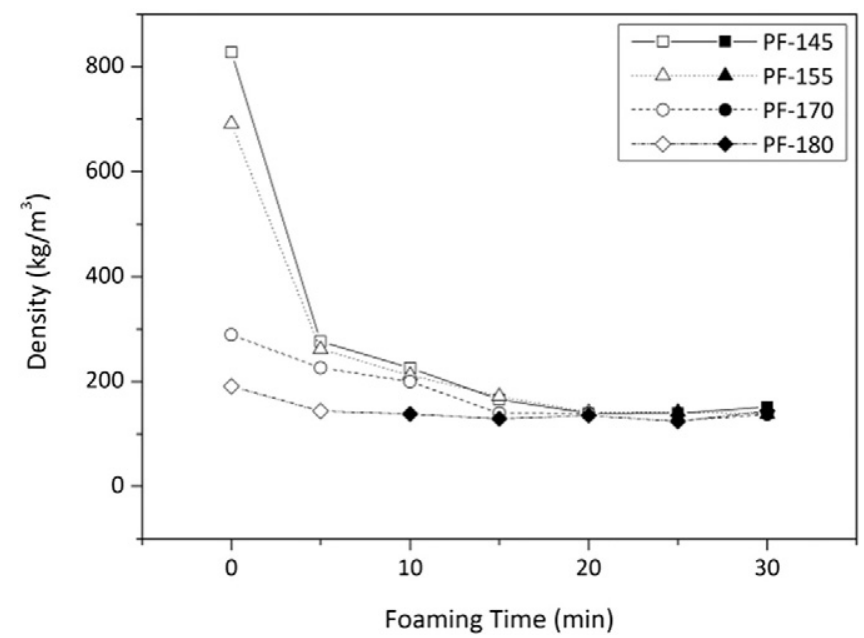

Fig. 5. Evolution of density. Open symbols correspond to growing stage and closed symbols to ageing stage.

considered as the optimum times for the production of foams $\left(t_{\min }\right)$, as they are the minimum times necessary to achieve the maximum expansion ratio.

\subsubsection{Cellular structure}

Fig. 6 represents the temporal evolution of the different structural parameters: number of cells in the image $\left(N_{2 D}\right)$, anisotropy ratio $(R)$ and orientation angle of the cells $(\theta)$. Growing and ageing stages are once again distinguished by open and closed symbols respectively.

As it has been aforementioned, all the different materials show a certain number of cells at foaming time equal to zero (Fig. 6.a). Two different tendencies can be appreciated in the evolution of the number of cells. For the series produced at low pre-foaming temperatures (PF145 and PF-155) the initial number of cells seems to be constant along both growing and ageing stages. The other two series (PF-170 and PF180) present a decrease of the number of cells during growing, whereas it remains practically constant during ageing. There is a relationship between the number of cells in the ageing stage and the pre-foaming temperature; the higher the temperature, the larger the number of cells. In order to explain these results two mechanisms must be taken into consideration: release of blowing agent and crosslinking. The influence of these two mechanisms in the number of cells will be discussed in the next section (Discussion section).

Fig. 6.b indicates that anisotropy ratios of the order of 2.5 and even higher have been obtained. The highest value, 3.75 , belongs to the series with the lowest pre-foaming temperature (PF-145), which presents the highest anisotropy ratio just at the moment of filling the mold. The next one corresponds to the series PF-155; however, series PF-170 and PF180 have in general lower anisotropy ratios. At a foaming time equal to zero, PF-145 and PF-155 show anisotropy ratios near to 1 (that is, they show an isotropic structure); nonetheless, PF-170 and PF-180 present anisotropy ratios higher than 2 as a consequence of the large expansion that they have already suffered during the pre-foaming step.

Regarding the orientation of the cells, they are highly orientated in the $y$ axis (orientation angle equal to $90^{\circ}$ ) as it can be seen in Fig. 6.c. This means that the majority of the cells have expanded in one direction, which explains the high anisotropy ratios obtained.

\subsection{Analysis of the optimum cellular materials}

\subsubsection{Density}

The foams produced at the so-called optimum times $\left(t_{\min }\right)$ have been chosen in order to further analyze the effect of the pre-foaming temperature. Densities of these foams are summarized in Table 2. 

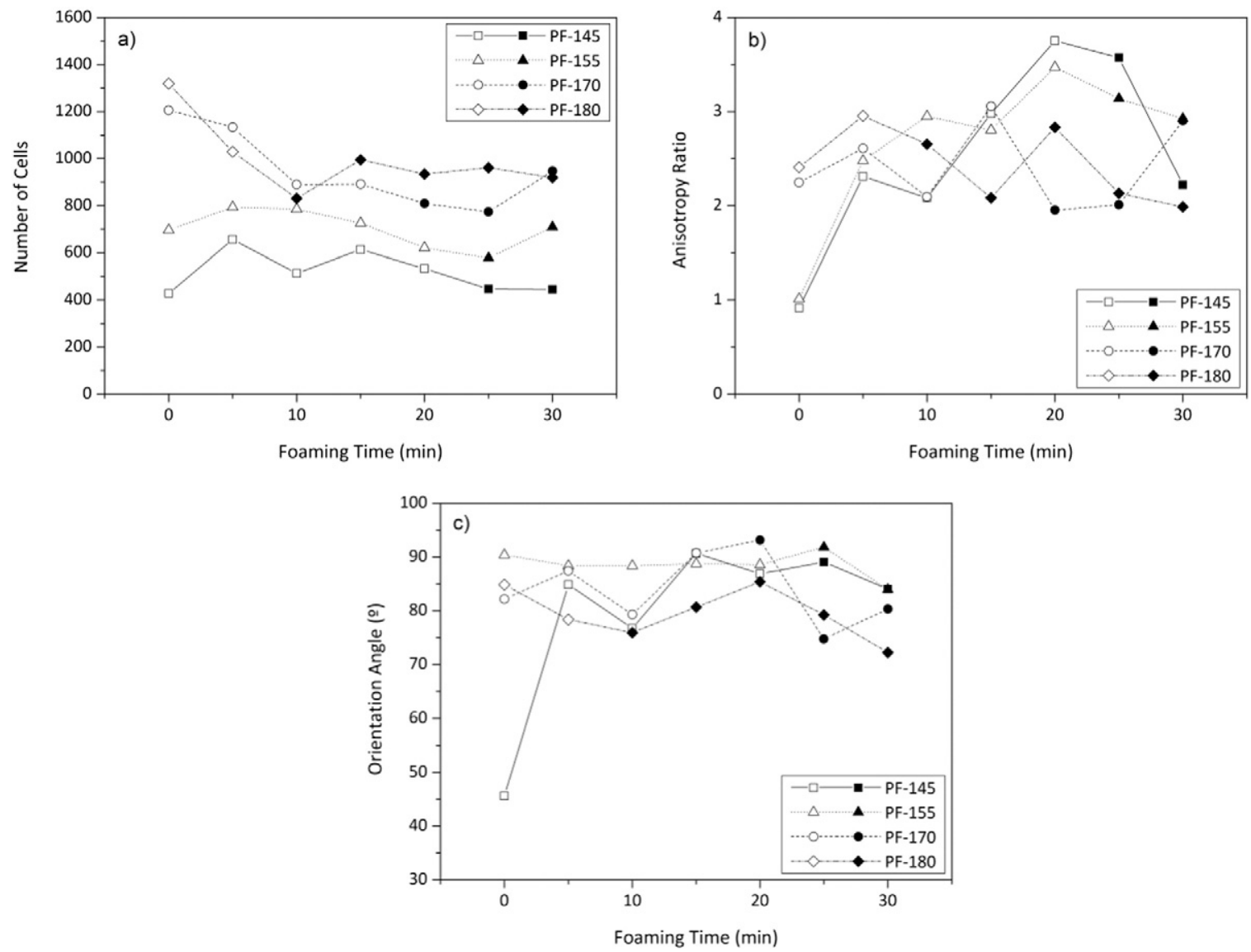

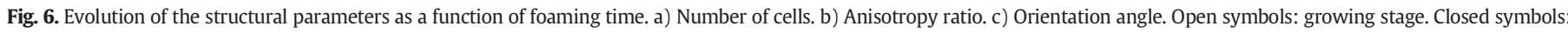
ageing stage.

The density is very similar for the four samples chosen, making the comparison possible.

\subsubsection{Cellular structure}

The cellular structure parameters obtained from the optimum foams are collected in Table 3. The number of cells in the image, $N_{2 D}$, depends on the pre-foaming temperature, in fact, $N_{2 D}$ increases as the prefoaming temperature increases. The cell density $N_{v}$, which is proportional to the total number of cells in the foam, presents the same behavior. As a consequence, the cell size $\phi_{3 D}$ varies as well with the prefoaming temperature, following an opposite behavior since this parameter decreases as the pre-foaming temperature increases. The cell size is strongly influenced by the cell density. In foams with similar densities, the cell size decreases as the cell density increases [16,23]. Experimental results indicate that the two-step foaming method presented in this work allows controlling the cell size of the final foam by changing the pre-foaming temperature.

The study of the optimum foams shows differences in the anisotropy ratios which were not observed as clearly in the complete kinetics. In particular, the data show a relation between the anisotropy ratio and the pre-foaming temperature. The anisotropy ratio decreases as the pre-foaming temperature increases (Fig. 7). The explanation of this phenomenon must take into account the behavior observed for the number of cells, which is opposite to that followed by the anisotropy ratio (Fig. 7). The expansion of each single cell in the $y$ direction, during the

Table 2

Density of the optimum foam of each series.

\begin{tabular}{lllll}
\hline Series & PF-145 & PF-155 & PF-170 & PF-180 \\
\hline$\rho\left(\mathrm{kg} / \mathrm{m}^{3}\right)$ & 138.9 & 141.2 & 139.8 & 143.6 \\
\hline
\end{tabular}

foaming step, is limited by the fact of having a large initial number of cells, which in turn is caused by high pre-foaming temperatures, resulting in low anisotropy ratios. When low pre-foaming temperatures are used fewer cells are created in this first step; therefore, cells are able to expand more freely in the $y$ direction leading to foams with higher anisotropy ratios.

Summarizing, the pre-foaming temperature controls the number of cells, which in turn controls the anisotropy ratio.

\subsubsection{Mechanical tests}

Mechanical tests in compression were performed on the optimum foams in the expansion direction ( $y$ direction). Table 4 shows the elastic modulus $(E)$ in this direction.

As it could be expected, the elastic modulus increases with the anisotropy ratio. It is possible to increase the elastic modulus 2.63 times by varying the pre-foaming temperature from $180{ }^{\circ} \mathrm{C}$ to $145^{\circ} \mathrm{C}$, that is, by varying the anisotropy ratio from 2.95 to 3.75 . Hence, the production route explained in this work allows obtaining foamed materials with the same density and different mechanical properties just by controlling the anisotropy ratio. Results obtained are in agreement with those found in literature $[2,22,32,36]$.

Table 3

Cellular structure parameters of the optimum foam of each series.

\begin{tabular}{lllll}
\hline Series & PF-145 & PF-155 & PF-170 & PF-180 \\
\hline$N_{2 D}$ & 533 & 621 & 891 & 1029 \\
$R$ & 3.75 & 3.47 & 3.06 & 2.95 \\
$N_{v}\left(\mathrm{~cm}^{-3}\right)$ & 4450.1 & 6732.8 & 9350.3 & $12,174.1$ \\
$\phi_{3 D}(\mu \mathrm{m})$ & 1238.6 & 960.5 & 848.3 & 817.3 \\
\hline
\end{tabular}




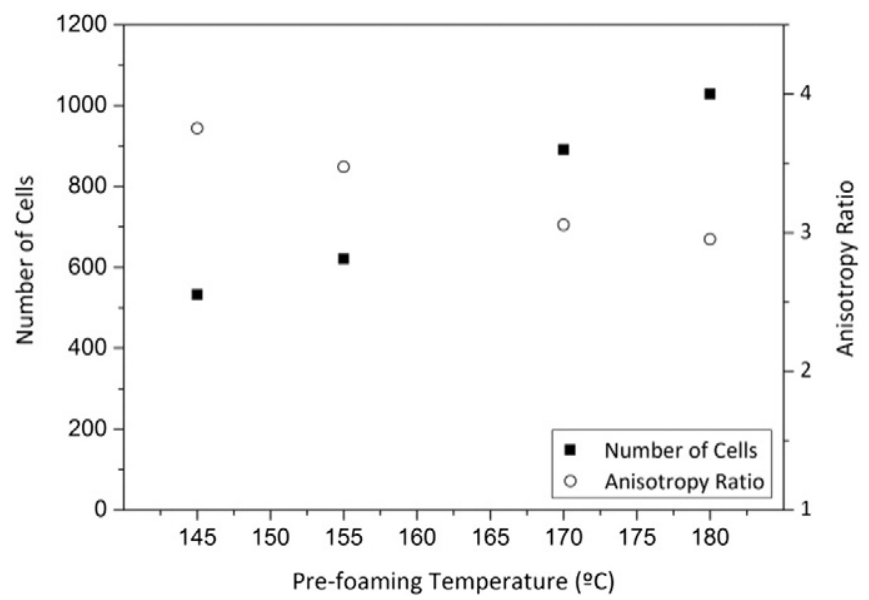

Fig. 7. Number of cells and anisotropy ratio as a function of the pre-foaming temperature.

\section{Discussion}

\subsection{Number of cells}

According to the classical nucleation theory (CNT) the homogeneous nucleation rate $(N)$ is expressed as indicated by Eq. (9) $[37,38]$.

$N=C \cdot f \cdot \exp \left(-\frac{\Delta G}{k_{B} T}\right)$

where $C$ is the concentration of gas molecules, $f$ is the frequency factor of the gas molecules, $k_{B}$ is the Boltzman's constant and $\Delta G$ is the Gibbs free energy barrier, which depends on the surface tension $(\gamma)$ and on the pressure drop of the gas/polymer solution $(\Delta p)$, as Eq. (10) indicates.

$\Delta G=\frac{16 \pi \gamma^{3}}{3 \Delta p^{2}}$

Therefore, the larger the surface tension, the lower the nucleation rate. However, a high pressure gradient provides a larger nucleation rate. Final nucleation density should be a balance of both contributions. In a two-step foaming method including a pre-foaming step in which crosslinking occurs, the two mechanisms must be taken into consideration. On the one hand, the crosslinking affects the surface tension and on the other hand, the release of the gas creates a pressure gradient [39-41].

It has been previously found that the number of cells present in a cross-section of the foam depends on the pre-foaming temperature. During the first step both crosslinking and release of gas take place simultaneously, but at different speeds depending on temperature. Table 5 presents the data of gel content and blowing agent decomposed (with respect to the total amount of it) at foaming time equal to zero.

Results indicate that high pre-foaming temperatures lead to a large amount of blowing agent decomposed. As a consequence an increase in the nucleation rate is produced, according to CNT, which results in a higher number of cells at the initial foaming time, as it is observed in Fig. 6.a.
Table 5

Blowing agent released after the pre-foaming step (foaming time $t=0 \mathrm{~min}$ ) and gel content after the pre-foaming step ( $t=0 \mathrm{~min}$ ) and at the end of the foaming process $(t=30 \mathrm{~min})$

\begin{tabular}{llll}
\hline Series & ADC decomposed (wt\%) $(t=0$ min $)$ & $\begin{array}{l}\text { Gel content }(\%) \\
(t=0 \text { min })\end{array}$ & $\begin{array}{l}\text { Gel content }(\%) \\
(t=30 \text { min })\end{array}$ \\
\hline PF-145 & 3.98 & $25 \pm 1$ & $83 \pm 2$ \\
PF-155 & 7.23 & $45 \pm 4$ & $84 \pm 2$ \\
PF-170 & 10.61 & $88 \pm 1$ & $81 \pm 2$ \\
PF-180 & 15.52 & $86 \pm 1$ & $82 \pm 3$ \\
\hline
\end{tabular}

For the PF-145 and the PF-155 series the number of cells remained constant along foaming time (Fig. 6.a). Crosslinking of the polymer matrix not only takes place during the pre-foaming step but also during foaming, as the results show (Table 5). As a consequence the surface tension of the polymer is increased. For this reason there is no additional creation of cells in the foaming process, as it is easier for the gas released by the ADC to fill the pores already formed than to create new ones. There are neither degeneration mechanisms which may reduce the number of cells as a result of the resistant crosslinked cell walls.

The PF-170 and the PF-180 series present a reduction of the number of cells during the growing stage; although, they are completely crosslinked from the initial foaming time (see Table 5). This reduction of the number of cells could be caused by cell coalescence. During the growing state a significant reduction in density is achieved (Fig. 5). This results in cells with very thin walls, which may break despite the crosslinking of the polymer matrix. Besides, as shown in Table 5 the amount of gas released at those moments is very high; therefore, there is a high pressure of gas inside the gas bubbles, which may induce cell wall ruptures as well. Cell coalescence is produced up to the moment at which cell walls reach a thickness enough to promote the stability of the cellular structure. After that moment, the number of cells remains constant (Fig. 6).

\subsection{Anisotropy ratio}

The relationship between the anisotropy ratio and the number of cells can be understood by studying the growing of the cells during the foaming process. The three dimensional cell size in both $x$ and $y$ axis for each series is depicted in Fig. 8. Again, growing and ageing stages are distinguished by different symbols.

Cell sizes in both directions increase during the growing stage and they remain almost constant during ageing. However, it is clear that the growth is much higher in the $y$ than in the $x$ direction, especially for PF-145 and PF-155. Therefore, it is possible to understand the system by assuming a one dimensional growing.

Taking this fact into consideration the scheme in Fig. 9 illustrates two general processes, in which the initial situation is isotropic (like PF-145 and PF-155) and the final one is anisotropic (Fig. 6.b). Situation A presents fewer cells than situation B. Assuming that the growing of the cells occurs only on the $y$ axis, the maximum possible anisotropy ratio is inversely proportional to the total number of cells. Thus, situation A presents a higher anisotropy ratio than situation $\mathrm{B}$, which is in agreement with the experimental results obtained in this work. In the case of PF-170 and PF-180, the initial situation is already anisotropic because the process has been stopped after some expansion had already taken place. In other words, an intermediate situation between the two states presented in Fig. 9 is obtained. Either way, this scheme shows that a lower initial number of cells allows reaching higher anisotropies, because cells have more space to grow in the vertical direction.

\section{Conclusions}

Highly anisotropic crosslinked HDPE foams produced by means of a two-step foaming method are studied in this work. Anisotropy ratios as high as 3.75 have been obtained. The foaming method proposed 

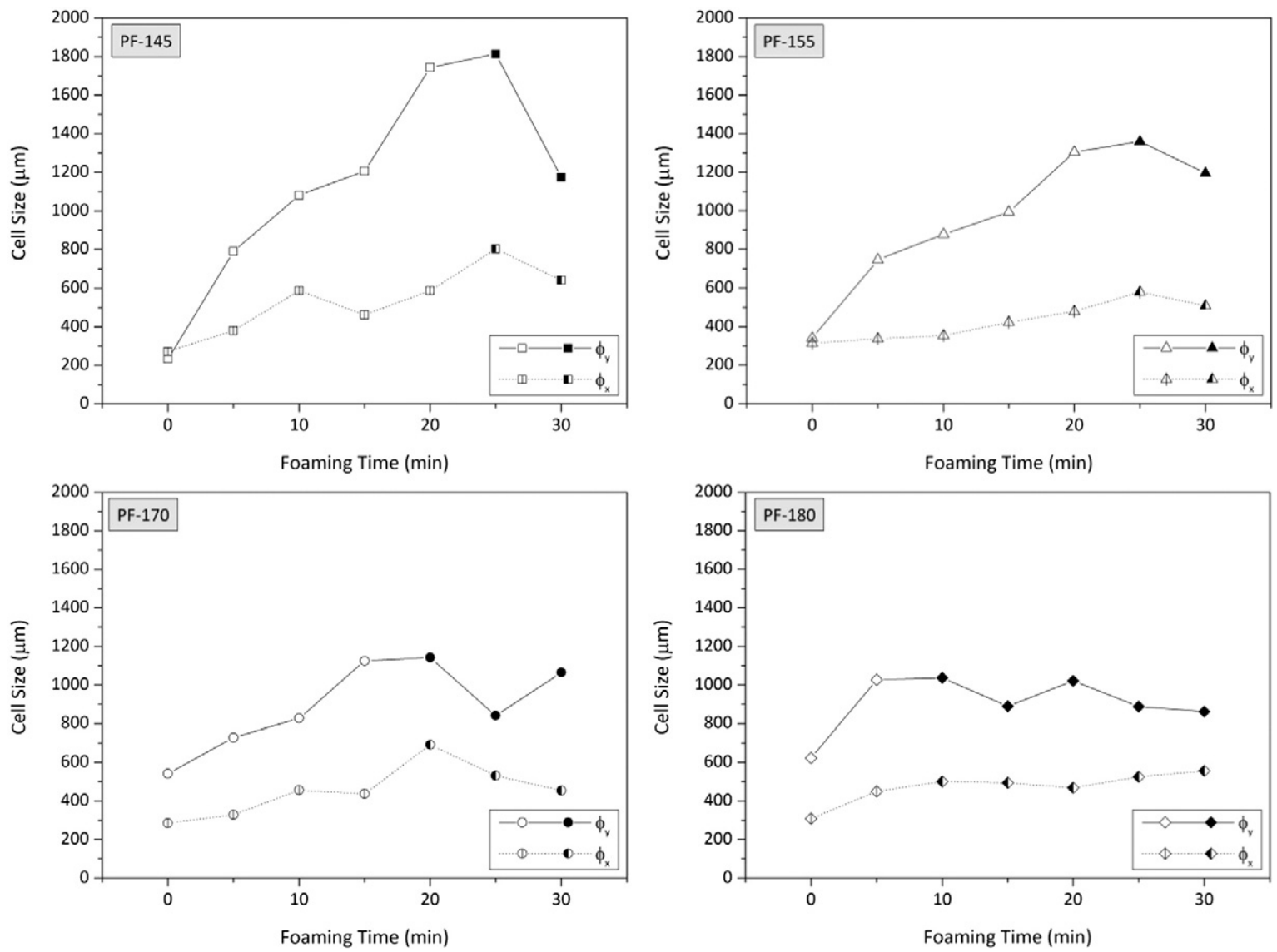

Fig. 8. Evolution of the cell size. Open symbols: growing stage. Closed symbols: ageing stage.

consists of a pre-foaming step and a foaming step. The effect of the prefoaming temperature was evaluated by producing foams using four different pre-foaming temperatures $\left(145,155,170\right.$ and $\left.180^{\circ} \mathrm{C}\right)$ and different foaming times (from 5 to $30 \mathrm{~min}$ ). Results show that a higher prefoaming temperature produces a larger number of cells in the foams. This is explained by applying classical nucleation theory: when the pre-foaming temperature increases the amount of blowing agent decomposes also increases, which leads to a higher nucleation rate.

\section{Initial}
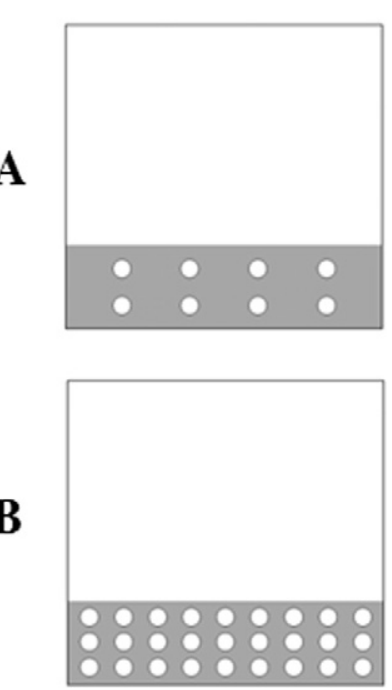

Final
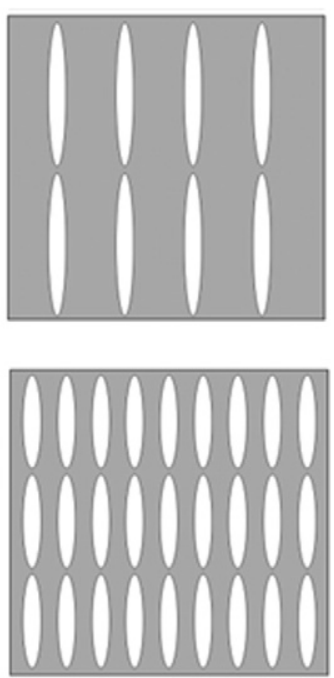

Fig. 9. Schematic representation of the expansion process for two different initial numbers of cells.
The optimum foam of each series was selected to perform a detailed study of the cellular structure parameters. The obtained results indicate that the anisotropy ratio decreases while increasing the pre-foaming temperature. This can be understood by taking into account the number of cells present in a cross-section of the foam. If a unidirectional growing is assumed, a larger number of cells will produce a lower anisotropy ratio and vice versa. Therefore, the pre-foaming temperature controls the number of cells, which in turns determines the final anisotropy of the foams. On the other hand mechanical tests in compression show an increase in the elastic modulus in the expansion direction while increasing the anisotropy ratio. In fact, an increase in the anisotropy ratio of 1.3 times leads to an increase in the elastic modulus of 2.6 times.

\section{Acknowledgments}

Financial support from FPU grant FPU14/02050 (V. Bernardo) from the Spanish Ministry of Education and from PIRTU contract (E. Laguna-Gutierrez) by Junta of Castile and Leon (EDU/289/2011) and cofinanced by the European Social Fund are gratefully acknowledged. Financial assistance from MINECO and FEDER program (MAT 2012 34901) MINECO, FEDER, UE (MAT2015-69234-R) and the Junta de Castile and Leon (VA035U13) are gratefully acknowledged.

\section{References}

[1] S.T. Lee, N.S. Ramesh, Polymeris Foams: Mechanisms and Materials, 2004

[2] D. Eaves, Handbook of Polymer Foams, Rapra Tech., 2004

[3] J. Zhang, M.F. Ashby, Mechanical selection of foams and honeycombs used for packaging and energy absorption, J. Mater. Sci. 29 (1994) 157-163.

[4] M. Avalle, G. Belingardi, R. Montanini, Characterization of polymeric structural foams under compressive impact loading by means of energy-absorption diagram, Int. J. Impact Eng. 25 (2001) 455-472.

[5] J. Salamone, Polymeric Materials Encyclopedia, Boca Raton, Florida, 1999. 
[6] M. Biron, Thermoplastics and Thermoplastic Composites - Technical Information for Plastics Users, Butterworth-Heinemann, Oxford, 2007.

[7] A.J. Peacock, Handbook of Polyethylene: Structure, Properties an Applications, Marcel Dekker, Inc, 2005

[8] P.R. Hornsby, Thermoplastics structural foams, Mater. Des. 3 (1982) 443-455.

[9] C. Tovar-Cisneros, R. Gonzalez-Nunez, D. Rodrigue, Effect of mold temperature on morphology and mechanical properties of injection molded HDPE structural foams, J. Cell. Plast. 44 (2008) 223-237.

[10] Y. Zhang, D. Rodrigue, A. Ait-Kadi, High density polyethylene foams. IV. Flexural and tensile moduli of structural foams, J. Appl. Polym. Sci. 90 (2003) 2139-2149.

[11] D.E. Ramirez-Arreola, C. Sedano de la Rosa, N.B. Haro-Mares, J.A. Ramirez-Moran, A.A. Perez-Fonseca, J.R. Robledo-Ortiz, Compressive strength study of cement mortars lightened with foamed HDPE nanocomposites, Mater. Des. 74 (2015) 119-124.

[12] L.J. Gibson, M.F. Ashby, Cellular Solids: Structure and Properties, Cambridge University Press, Cambridge, 1997.

[13] R. Gendron, Thermoplastic Foam Processing, CRC Press, 2004.

[14] C. Saiz-Arroyo, M.A. Rodriguez-Perez, J. Tirado, A. López-Gil, J.A. de Saja, Structureproperty relationships of medium-density polypropylene foams, Polym. Int. 62 (2013) 1324-1333.

[15] S.T. Lee, C.B. Park, N.S. Ramesh, Polymeric Foams: Science and Technology, CRC Press, Boca Raton, 2007.

[16] W. Zhai, J. Yu, L. Wu, W. Ma, J. He, Heterogeneous nucleation uniformizing cell size distribution in microcellular nanocomposites foams, Polymer 47 (2006) 7580-7589.

[17] J.E. Weller, V. Kumar, Solid-State Microcellular Polycarbonate Foams II, The Effect of Cell Size on Tensile Properties, Polymer Engineering \& Science, 2010.

[18] J. Andersons, M. Kirpluks, L. Stiebra, U. Cabulis, Anisotropy of the stiffness and strength of rigid low-density closed-cell polyisocyanurate foams, Mater. Des. 92 (2016) 836-845.

[19] N. Mills, Polymer Foams Handbook, Elsevier, 2007.

[20] D. Klempner, K.C. Frisch, Handbook of Polymeric Foams and Foam Technology, 1991.

[21] Y. Zhang, D. Rodrigue, A. Ait-Kadi, High density polyethylene foams. II. Elastic modulus, J. Appl. Polym. Sci. 90 (2003) 2120-2129.

[22] A.T. Huber, L.J. Gibson, Anisotropy of foams, J. Mater. Sci. 23 (1988) 3031-3040.

[23] B. Depreitere, J. Goffin, C. Van Lierde, B. Haex, J. Vander Sloten, R. Van Audekercke, G. Van der Perre, I. Verpoest, P. Verschueren, H. Delye, Protective Helmet, 2008 (Patent WO 2006/005143)

[24] K. Vandenbosche, J. Ivens, I. Verpoest, J. Goffin, G.V.D. Perre, M.A. Rodriguez-perez, A novel way of producing protective anisotropic foams with complex shapes, Foams 2012, p. 2012.

[25] K. VandenBosche, I. Verpoest, J. Ivens, M.A. Rodriguez-Perez, Complexly Shaped Anisotropic Foam Manufacturing, 2013 (Patent WO 2013030318 A1).
[26] A. Lopez-Gil, J. Escudero, E. Laguna-Gutierrez, C. Saiz-Arroyo, M. Rodriguez-Perez, A. Anicell, Low density and non-crosslinked polypropylene foams as a promising option to produce structural panels, Eurotec, 2013, 2013, pp. 374-379.

[27] J. Escudero, A. Lopez-Gil, E. Laguna-Gutierrez, M. Rodriguez-Perez, A. Low density non-crosslinked closed-open cell polypropylene foams with high mechanical properties.pdf, Cell. Polym. 35 (2016) 101-118.

[28] B. Wicklein, A. Kocjan, G. Salazar-Alvarez, F. Carosio, G. Camino, M. Antonietti, L. Bergström, Thermally insulating and fire-retardant lightweight anisotropic foams based on nanocellulose and graphene oxide, Nat. Nanotechnol. 10 (2014) 277-283.

[29] S. Faraji, K.L. Stano, O. Yildiz, A. Li, Y.T. Zhu, P.D. Bradford, Ultralight anisotropic foams from layered aligned carbon nanotube sheets, Nanoscale 7 (2015) 17038-17047.

[30] E. Laguna-Gutierrez, R. Van Hooghten, P. Moldenaers, M.A. Rodriguez-Perez, Understanding the foamability and mechanical properties of foamed polypropylene blends by using extensional rheology, J. Appl. Polym. Sci. 132 (2015) 42430(1)-42430(14).

[31] K.A. Arora, A.J. Lesser, T.J. McCarthy, Preparation and characterization of microcellular polystyrene foams processed in supercritical $\mathrm{CO}_{2}$, Macromolecules 38 (1997) 446-447.

[32] L. Oliveira-Salmazo, A. Lopez-Gil, F. Silva-Bellucci, A.E. Job, M.A. Rodriguez-Perez, Natural rubber foams with anisotropic cellular structures: mechanical properties and modeling, Ind. Crop. Prod. 80 (2016) 26-35.

[33] F.G. Kleiner, L. Radojewski, L. Mühlbauer, K.-H. Müller, Laminates of Polyethylene Foam with Anisotropic Pore Structure, 1987.

[34] J. Pinto, E. Solo, M.A. Rodriguez-perez, J.A.D. Saja, Characterization of the cellular structure based on user-interactive image analysis procedures, J. Cell. Plast. 49 (2013) 555-575.

[35] V. Kumar, Process Synthesis for Manufacturing Microcellular Thermoplastic Parts, Massachusetts Institute of Technology, 1988

[36] B.S. Mehta, E.A. Colombo, Mechanical properties of foamed thermoplastics, J. Cell. Plast. 12 (1976) 59-66.

[37] V. Mittal, Polymer Nanocomposite Foams, CRC Press, 2014.

[38] V.I. Kalikmanov, Nucleation Theory, Springer, 2013.

[39] D.G. Legrand, G.L. Gaines, The molecular weight dependence of polymer surface tension, J. Colloid Interface Sci. 31 (1969) 162-167.

[40] C.I. Poser, I.C. Sanchez, Surface tension theory of pure liquids and polymer melts, J. Colloid Interface Sci. 69 (1979) 539-548.

[41] A.W. Adamson, A.P. Gast, Physical Chemistry of Surfaces, 1997 